\title{
Upper Limit of Vulnerability for Induction of Ventricular Fibrillation
}

National Cancer Institute

\section{Source}

National Cancer Institute. Upper Limit of Vulnerability for Induction of Ventricular

Fibrillation. NCI Thesaurus. Code C101237.

Stimulus strength above which electrical stimulation from a cardiac device cannot induce ventricular fibrillation even when the stimulus occurs during the vulnerable period of the cardiac cycle. (ACC) 